\title{
A Study of the Yield and Post-Yield Behavior of Syndiotactic Polypropylene
}

\author{
Claudia Marano, Nadia Perillo, Marta Rink \\ Dipartimento di Chimica, Materiali e Ingegneria Chimica “Giulio Natta”, Piazza Leonardo da Vinci 32, 20133 Milano, Italia \\ Correspondence to: C. Marano (E-mail: claudia.marano@polimi.it)
}

Received 10 June 2014; revised 11 July 2014; accepted 11 July 2014; published online 31 July 2014

\begin{abstract}
The strain recovery of three syndiotactic polypropylenes (s-PPs) differing in the percentage of [rrrr] pentad is investigated. A suitable method based on loading-unloading tests at constant displacement rate in tensile loading conditions is adopted to measure the residual and recovered strain components of the applied strain. The method allows to obtain a large amount of data from few tests and to explore a wide strain range. The dependence of the material's strain recovery
\end{abstract}

INTRODUCTION Syndiotactic polypropylene (s-PP) has been widely studied in the last fifty years mainly concerning its crystal polymorphism and morphology (see e.g., refs. 1-3) and the effects of drawing on material structure. ${ }^{2-6}$ When slowly cooled from the melt state or when crystallized from solution, s-PP shows a crystalline phase consisting of an antichiral packing (right-handed and left-handed chains alternating along $a$ and $b$ axis of the unit cell) in which the macromolecular chains show a twofold helical conformation in an orthorhombic crystalline cell. This crystalline form, which is referred to as form I, transforms into the trans-planar conformation-arranged form III when material is strained. Form III is a metastable form of s-PP, characterized by an orthorhombic crystalline cell. The strain value at which the onset of the transformation of form I into form III occurs depends on the syndiotactic pentad [rrrr] content. ${ }^{5}$ The appearance of a mesophase in place of form III or previous to form III formation is also controlled by the syndiotactic pentad [rrrr] content. ${ }^{5}$

When the material is annealed under stretching ${ }^{2}$ or is unloaded,$^{5}$ form III transforms into the helical conformationarranged form II, characterized by a C-center structure in which the macromolecular chains are packed with the same chirality. If the mesophase forms under stretching, the crystalline form I is present after unloading.

Several studies have been carried out also to analyze and interpret the peculiar "elastic" behavior of s-PP: $:^{5,7-9}$ when s-PP is drawn beyond yield and then unloaded the applied on the applied strain is analyzed in relation to s-PP straininduced microstructural changes and crystalline form transitions, which are reported in literature.

KEYWORDS: crystal structures; poly(propylene) (PP); structureproperty relations; syndiotactic; yielding

strain partially recovers and, if reloaded within the recovered strain range and then again unloaded, it shows practically no residual strain. It thus behaves differently from the so-called "Hard elastic fibers" or "Springy polymers,"10,11 whose elastic behavior is related to a particular material morphology which is induced during processing. The elastic behavior of s-PP has been related to the reversible crystalcrystal phase transition between the trans planar form III and the helical form II or I, ${ }^{12}$ while other authors ${ }^{7-9}$ report that molecules' conformational transitions from trans planar to helical conformation, both within and between crystals, are responsible for the observed elastic behavior.

A very significant advance in the study of yield and postyield behavior of semicrystalline polymers has been achieved by Strobl and coworkers ${ }^{6,13,14}$ performing cyclic tensile tests, using a video controlled tensile testing machine. The adopted experimental method allowed determining true stress-strain curves at constant strain rates, overcoming the problem of the neck formation. Moreover, the cyclic test allowed to measure the residual and recovered strain components of the applied strain and how they change as strain increases. Irrespective of the type of polymer, it was found that the material's "differential compliance," the strain dependence of the residual or recovered strain, and the material's crystalline microstructure simultaneously change at four strain values which are characteristic of the material, being affected neither by its crystallinity degree nor by the temperature. Further, different deformation processes, which are progressively activated at these strain levels, have been identified. 
TABLE 1 Characteristic Data of s-PPs

\begin{tabular}{llllcc}
\hline Material & $M_{\mathrm{w}}\left(10^{3} \mathrm{~g} / \mathrm{mole}\right)$ & {$[\mathrm{rrrr}](\%)$} & $T_{\mathrm{g}}\left({ }^{\circ} \mathrm{C}\right)$ & $T_{\mathrm{m}}\left({ }^{\circ} \mathrm{C}\right)$ & 160 \\
s-PP91.5 & 766,000 & 91.5 & 0 & 125 \\
s-PP78 & 193,000 & 78 & 0 & 26 \\
s-PP54 & $1,300,000$ & 54 & $0^{\mathrm{b}}$ & $66^{\mathrm{b}}$
\end{tabular}

a $\chi=\frac{\Delta H_{m}}{\Delta H^{0}}$, where $\Delta H_{\mathrm{m}}$ is the experimental melting enthalpy and $\Delta H^{0}=183 \frac{\mathrm{J}}{\mathrm{g}}$ is s-PP melting enthalpy for $100 \%$ of crystallinity. ${ }^{6}$

${ }^{\mathrm{b}}$ Data measured four days after molding.

They have been described as isolated inter and intralamellae slip processes, collective slip processes, fragmentation of crystallites, and chain disentanglements, respectively. Further works ${ }^{15-17}$ have examined the deformation mechanisms in semicrystalline polymers. In particular, the relative role of the amorphous phase and of the crystalline blocks are discussed ${ }^{15,16}$ and the conversion of lamellar crystals into crystalline fibrils is described as a melting-recrystallization process. $^{15,17}$

Furthermore according to the work by Men et al. ${ }^{15}$ this process occurs when the stress transferred by the amorphous stretched phase to the crystallites reaches a critical value.

In the present work, loading-unloading tests were performed on three s-PPs, differing in syndiotactic pentad [rrrr] content. The residual and the recovered strain components of the overall strain were determined as a function of the applied strain for all the materials. A proper test method was set up to obtain true stress-strain curves using standard

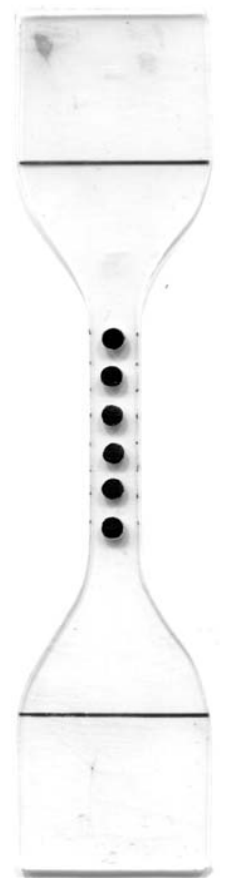

FIGURE 1 Sample for tensile test: black marks were placed for strain measurement with video-extensometer.

dumb-bell specimens also for materials which neck at yielding. This method reduced significantly the number of specimens to be tested, and allowed to explore a wide range of strains.

\section{EXPERIMENTAL}

\section{Materials}

Three commercial s-PPs, differing in syndiotactic pentad [rrrr] content, were studied in this work. The materials were kindly supplied by Atofina, who provided their structural characteristics, reported in Table 1. The material's code reflects its [rrrr] pentad content. Materials thermal properties (glass transition temperature, $T_{\mathrm{g}}$, melting temperature, $T_{\mathrm{m}}$, and crystallinity degree, $\chi$ ), measured by differential scanning calorymetry tests performed at $10{ }^{\circ} \mathrm{C} / \mathrm{min}$ heating rate, are also reported in Table 1 . The data relevant to $\mathrm{s}^{-}$ PP54 were measured 4 days after molding, when a constant value of crystallinity has been reached in the material, which crystallizes slowly at room temperature.

\section{Test Specimens}

s-PP plates $1 \mathrm{~mm}$ thick were obtained by compression molding $\left(0.2 \mathrm{MPa}\right.$ and $180{ }^{\circ} \mathrm{C}, 165{ }^{\circ} \mathrm{C}$, and $140{ }^{\circ} \mathrm{C}$ for s-PP91.5, s-PP78, and s-PP54, respectively). The plates were slowly cooled to room temperature. Dumb-bell specimens, with a gauge length of $33 \mathrm{~mm}$, were obtained by die cutting. Black marks were placed along the specimen gauge length, as

(a)

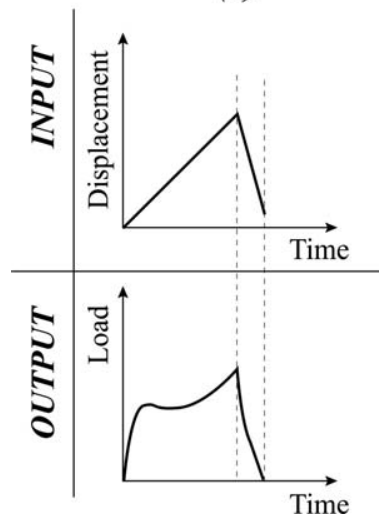

FIGURE 2 Sketch of a single-cycle (a) and multi-cycles (b) loading-unloading tensile tests. 


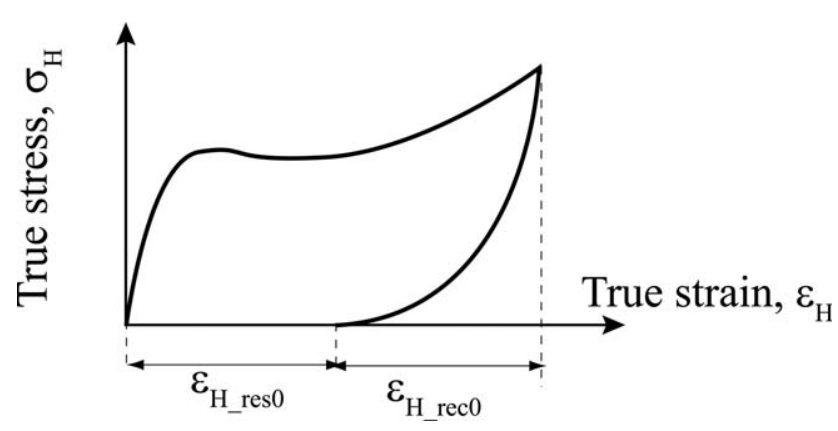

FIGURE 3 Definition of residual and recovered strain components of the overall true strain at the end of unloading.

shown in Figure 1, to measure the local deformation using a video-extensometer.

Thus, measuring the distance between the marks delimiting the generic $i$-Zone, the local true strain, $\epsilon_{H_{i}}=\ln \lambda_{i}=\ln \frac{l_{i}}{l_{i_{0}}}$, was measured in every Zone of the specimen and the true stress, $\sigma_{\mathrm{T}_{i}}=\frac{P}{A_{i_{0}}} \lambda_{i}$, determined ( $P$ is the applied load, $A_{i 0}$ is the unstrained specimen cross-section, measured between two marks initially at a distance $l_{i 0}$ and $l_{i}$ is their actual distance during the test).

\section{Mechanical Tests}

Uniaxial tensile loading-unloading tests were performed, at room temperature, on a displacement-controlled dynamometer (Instron 1121) at constant displacement rate. Both single-cycle and multi-cycles tests were carried out, as sketched in Figure 2.

Displacement rates of 5 and $50 \mathrm{~mm} / \mathrm{min}$ were applied in both the loading and the unloading steps.

Residual $\left(\varepsilon_{H_{\mathrm{res}} 0}\right)$ and recovered $\left(\varepsilon_{H_{\mathrm{rec}} 0}\right)$ strain components of the overall true strain, $\varepsilon_{H}$, defined as in Figure 3, were measured for each value of the applied strain after unloading.

\section{Testing Method}

In this work, a simple test method was properly set up to analyze the material's strain recovery capability as a function of the applied strain. This method simplifies the experimental work by reducing significantly the number of specimens required. The method is sound when strain localization and necking take place. In this case, during a tensile test, the deformation is not homogeneous and different regions along the gauge length reach different strains at the same loading time. If the distance between two successive marks reported on a dumb-bell specimen (Fig. 1) is quite small, the specimen deformation within a "Zone" can be supposed to be homogeneous. Nevertheless, the different Zones will undergo different strain histories [see for e.g., Fig. 4(a) reporting the true strain measured in two Zones of a s-PP78 specimen drawn at a displacement rate of $5 \mathrm{~mm} / \mathrm{min}$ up to a nominal strain of $600 \%]$.

The different Zones can be considered as different test specimens provided the mechanical behavior is the same irrespective of the strain history. For example, this was verified for the two Zones in Figure 4(a), as shown by the good superposition of the two relevant stress-strain curves in Figure 4(b). As much as this hypothesis is valid, when a dumb-bell specimen is loaded up to a given overall strain and then unloaded the $n_{i}$ Zones along the gauge length can be considered as $n_{i}$ homogeneously deformed specimens drawn up to different strains and then unloaded.

\section{RESULTS AND DISCUSSION}

Some preliminary tests were performed to verify the applicability of the proposed test method for the three materials, that is the material's response is the same irrespective of the different strain histories applied in the different Zones of the specimen.

In Figure 5, the strain measured during a monotonic loading tensile test at $5 \mathrm{~mm} / \mathrm{min}$ in different Zones of a single specimen's gauge length is reported versus time for s-PP54 (a), s-PP78 (b), and s-PP91.5 (c) respectively. It can be observed that the deformation phenomenology is similar for s-PP78 and s-PP91.5: at first all the Zones are homogeneously drawn, then the strain mainly localizes in a single Zone, which rapidly deforms and reaches a plateau strain value
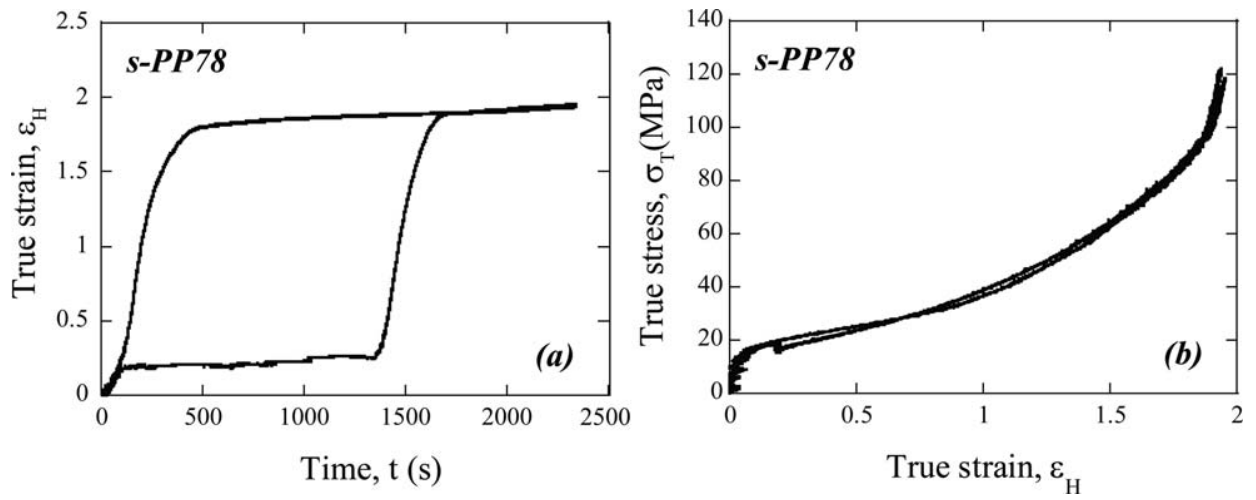

FIGURE 4 True strain vs. loading time measured in two different zones of a single s-PP78 dumb-bell specimen (a) and the relevant true stress-strain curves (b). Displacement rate $5 \mathrm{~mm} / \mathrm{min}$. 

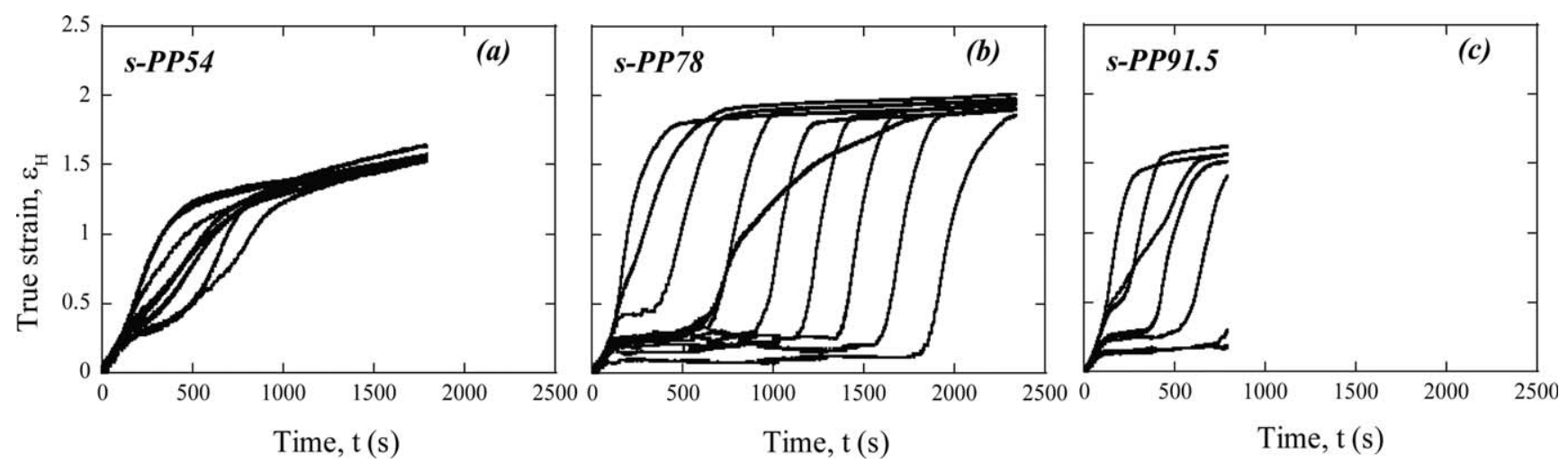

FIGURE 5 Time dependence of the true strain measured in different zones of a single specimen of s-PP54 (a), s-PP78 (b), and sPP91.5 (c). Displacement rate $5 \mathrm{~mm} / \mathrm{min}$.

after which it is not further strained and another specimen's Zone starts to rapidly deform. For s-PP54, instead, the deformation results to be less localized: after an initial homogeneous strain of the whole gauge length, all the Zones are drawn but each at a different drawing rate, reaching a strain of about 1.4. Then the sample deforms almost homogeneously. In Figure 6, the true stress-strain curves relevant to the different Zones of a single specimen are reported for sPP54 (a), s-PP78 (b), and s-PP91.5 (c), respectively: it can be observed that for the three materials the stress-strain response during loading is not affected by the applied drawing history.

To check that the deformation history does not affect the stress-strain response during unloading too, single cycle loading-unloading tests differing in the displacement rate of the unloading step were performed. In Figure 7, the true stress-true strain curves of s-PP54 are reported as an example: it can be observed that the material behavior in the unloading step is not affected by the displacement rate. Similar results were obtained also for s-PP78 and s-PP91.5. The obtained results indicate that the testing method previously described can be applied for a single loading-unloading test for the three materials, that is different Zones of a single specimen can be considered as different specimens drawn up to different strain values. Figure 8 reports the true stress-strain curves relevant to three Zones of a single specimen of s-PP54 (a), s-PP78 (b), and s-PP91.5 (c) under a multi-cycle loading-unloading tensile test performed at $5 \mathrm{~mm} / \mathrm{min}$. Curves relevant to monotonic loading up to failure are reported for comparison. For s-PP91.5 and s-PP78, the stress-strain curve of each loading step in the multicycles loading-unloading test overlaps with the stress-strain curve of the monotonic loading test for strain higher than the maximum strain reached in the previous cycle. Moreover it can be observed that material strain recovery in the unloading step and the subsequent loading curve result to be the same irrespective of the number of the applied loading-unloading cycles (see e.g., $\varepsilon_{H}=0.85$ for s-PP78). Thus for s-PP91.5 and s-PP78, successive loading-unloading cycles on a single specimen give the same information as many single cycle tests.

For s-PP54, instead [Fig. 8(a)] it can be observed that in the multi-cycle loading history, when the sample is reloaded, the material shows a different behavior with respect to that of the monotonically loaded material. Therefore for s-PP54 only single cycle tests were performed.

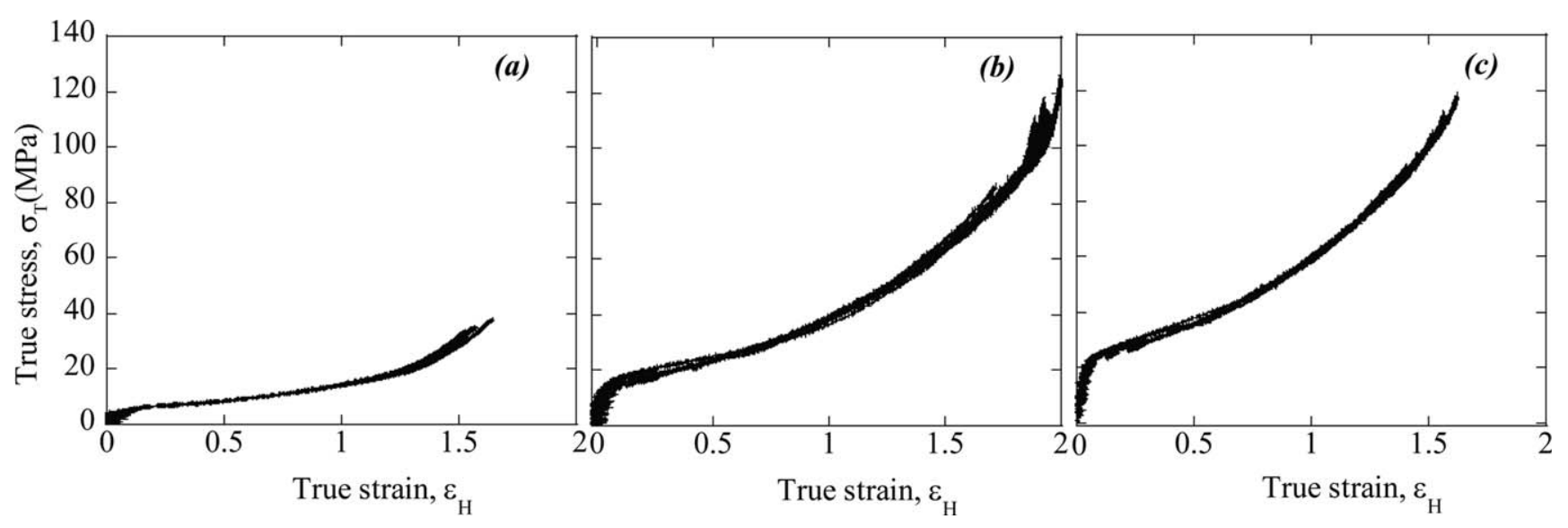

FIGURE 6 True stress-strain curves for s-PP54 (a), s-PP78 (b), and s-PP91.5 (c). Displacement rate $5 \mathrm{~mm} / \mathrm{min}$. 


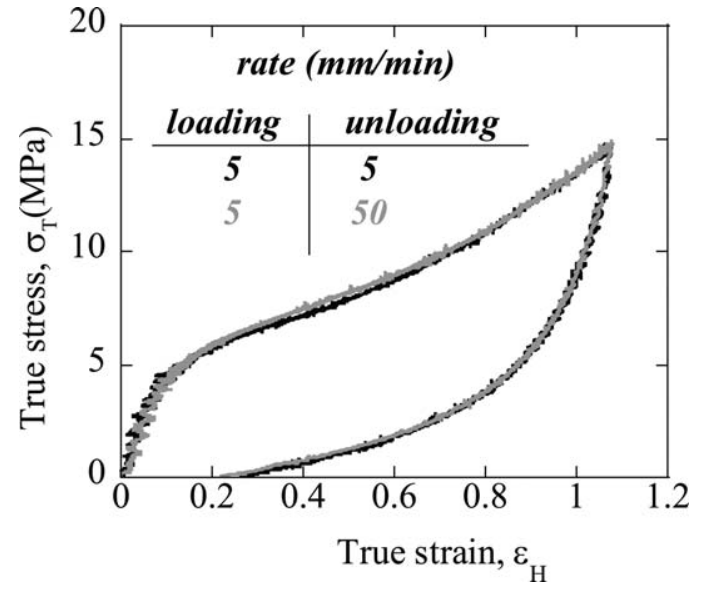

FIGURE 7 True stress-strain curves of s-PP54 in two singlecycle tests unloaded at different displacement rates.

From the multi-cycle tests performed on s-PP78 and sPP91.5 and from the single-cycle tests performed on the three materials, the residual, $\varepsilon_{H_{\mathrm{res}} 0}$ and the recovered, $\varepsilon_{H_{\mathrm{rec}}} 0$, strain components of the applied strain, $\varepsilon_{H}$, at the end of the unloading (defined in Fig. 2) were measured. Figure 9 reports $\varepsilon_{H_{\mathrm{res}} 0}$ and $\varepsilon_{H_{\mathrm{rec}} 0}$ versus $\varepsilon_{H}$ for s-PP54 (a), s-PP78 (b), and s-PP91.5 (c), respectively.

It can be observed that: (i) s-PP78 and s-PP91.5 behave similarly [Fig. $9(\mathrm{~b}, \mathrm{c})$ ] and for both the materials $\varepsilon_{H_{\mathrm{res}} 0}$ is slightly lower than $\varepsilon_{H_{\text {rec }} 0}$ up to an applied strain level of 0.4 ; at higher strains, the residual strain component is significantly higher than the recovered one; in s-PP54 [Fig. 9(a)], instead, $\varepsilon_{H_{\text {rec }} 0}$ is higher than the residual strain component over the whole explored strain range; (ii) changes in the trend of both $\varepsilon_{H_{\text {rec }} 0}$ and $\varepsilon_{H_{\text {res }} 0}$ vs. applied strain can be observed for all the materials: for s-PP78 and s-PP91.5 [Fig. 9(b,c)] first $\varepsilon_{H_{\text {res }} 0}$ increases fairly linearly with the applied strain up to $\varepsilon_{H}=0.4$ after which it still increases but with a higher slope. At $\varepsilon_{H}=1.8$ and $\varepsilon_{H}=1.4$ for s-PP78 and s-PP91.5 respectively, $\varepsilon_{H_{\text {rec }} 0}$ becomes constant and consequently the slope of the residual strain component vs. applied strain becomes unity, suggesting that the further deformation of the materials is completely plastic. This can be explained by the achievement at these strain levels of the maximum polymeric
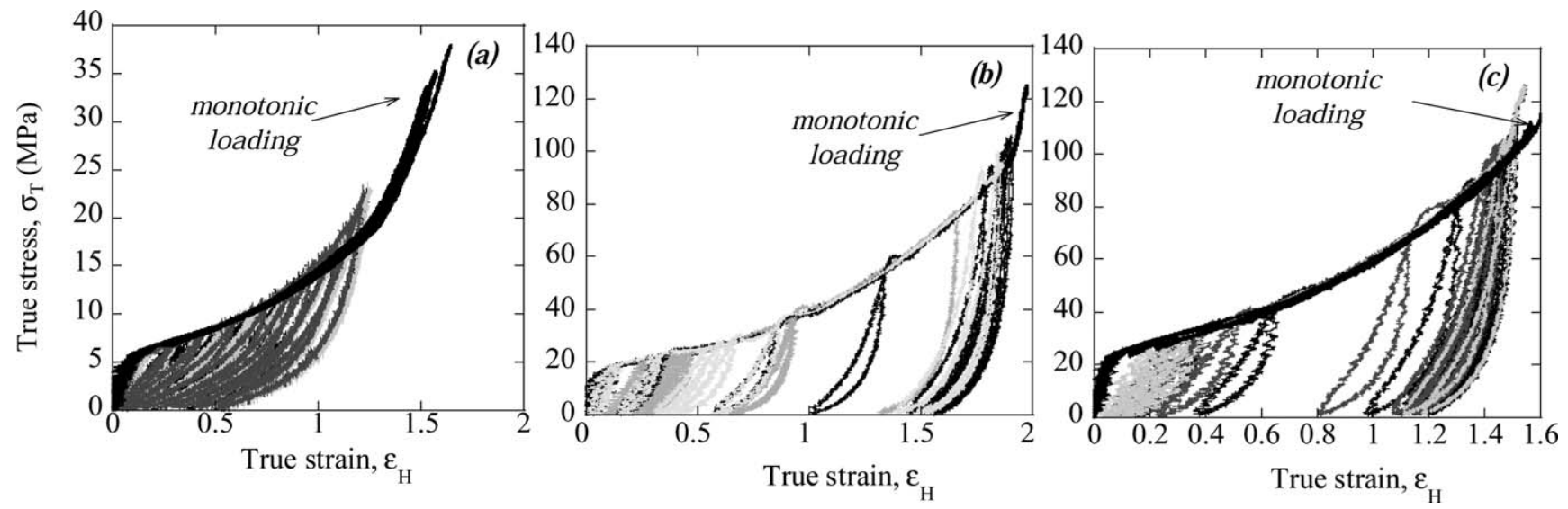

FIGURE 8 True stress-strain curves in monotonic loading and multi-cycles loading-unloading tests for s-PP54 (a), s-PP78 (b), and s-PP91.5 (c). Loading and unloading at $5 \mathrm{~mm} / \mathrm{min}$.
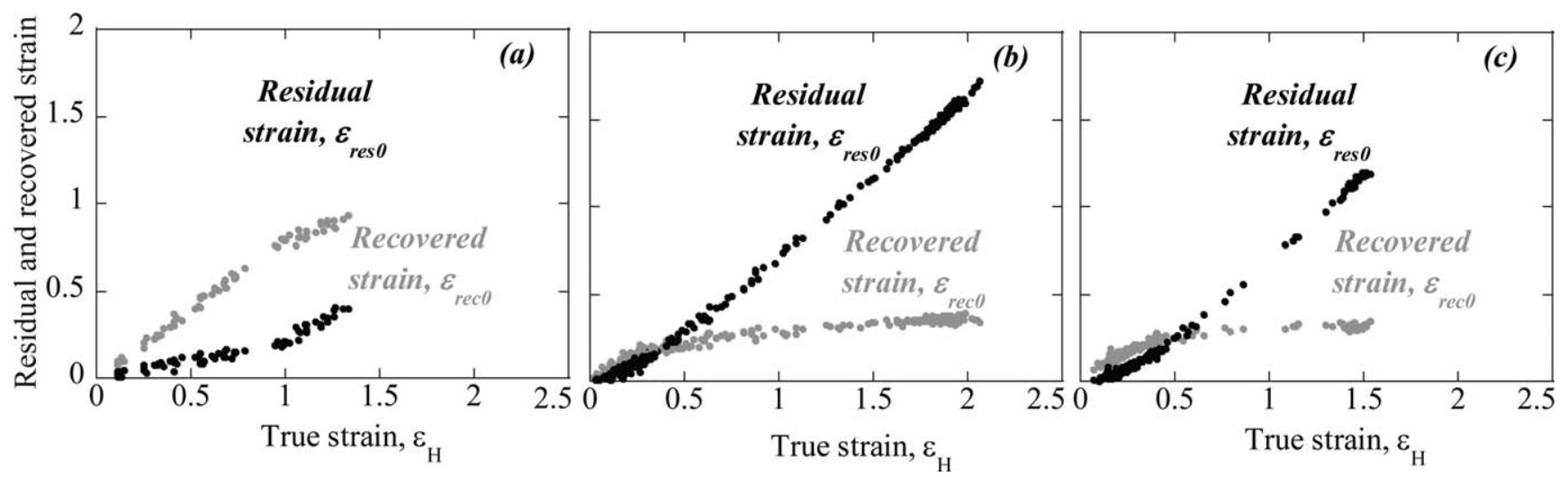

FIGURE 9 Dependence of the residual and recovered true strain components on the applied true strain for s-PP54 (a), s-PP78 (b), and s-PP91.5 (c). 
chains extension. In the case of s-PP54 [Fig. 9(a)] $\varepsilon_{H_{\text {res }} 0}$ as function of applied strain shows a bilinear trend with a change in slope at about $\varepsilon_{H}=1$.

An attempt was made to correlate the strains at which changes in the trends of recovered/residual strain components vs. applied strain occur with the strains at which microstructural or crystalline forms transitions take place.

For the three materials the first change in the trend of both $\varepsilon_{H_{\text {res }} 0}$ and $\varepsilon_{H_{\text {rec }} 0}$ occurs at a residual strain of $\sim 0.2$ as shown in Figure 10. This strain value is in good agreement with the "critical plastic true strain" reported in literature ${ }^{4}$ at which the conversion of lamellar crystals into crystalline fibrils occurs and a change in the slope of the residual strain vs. total applied strain takes place. In Figure 11 the data by Deplace et al. ${ }^{4}$ are shown together with the data from Men and Strobl ${ }^{6}$ which again show a bilinear trend with a change in slope at a residual strain of 0.2 . The total applied strain relevant to this residual strain is 0.4 for s-PP78 and s-PP91.5 and 1 for s-PP54. Such a shift in the overall strain at which fibrils start to form with crystallinity degree has been previously observed by Sun et al. ${ }^{16}$ on two different ethylene-octene copolymers. The authors attribute the shift to a different structure of the crystalline phase in the two copolymers. In the more crystalline copolymer a macroscopic crystalline rigid network interpenetrated with an entangled amorphous network is present while, in the less crystalline one, crystalline lamellar stacks are only weakly coupled through the amorphous entangled phase which causes an inhomogeneous strain distribution during deformation. It can therefore be thought that for S-PP78 and S-PP91.5, an interpenetrated network structure is present while for s-PP54 lamellar blocks tend to constitute a dispersed phase in an amorphous entangled matrix.

Regarding the strain-induced crystalline form transitions, it may be observed that for s-PP54 the critical plastic strain of 0.2 occurs at $\varepsilon_{H}=1$ at which the onset of the form I to mesoform transition takes place, as calculated from data reported by Auriemma and De Rosa ${ }^{5}$ even if for s-PP78 no change in the trend of the residual strain component vs.

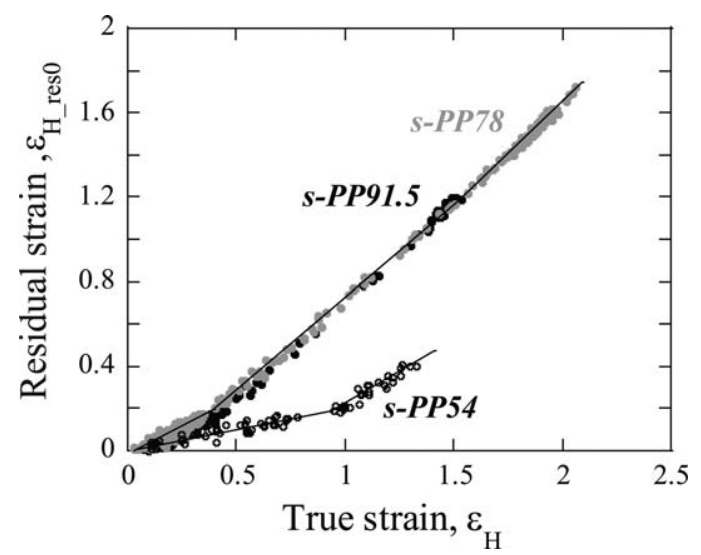

FIGURE 10 Dependence of the residual strain component on the applied true strain for s-PP54, s-PP78, and s-PP91.5.

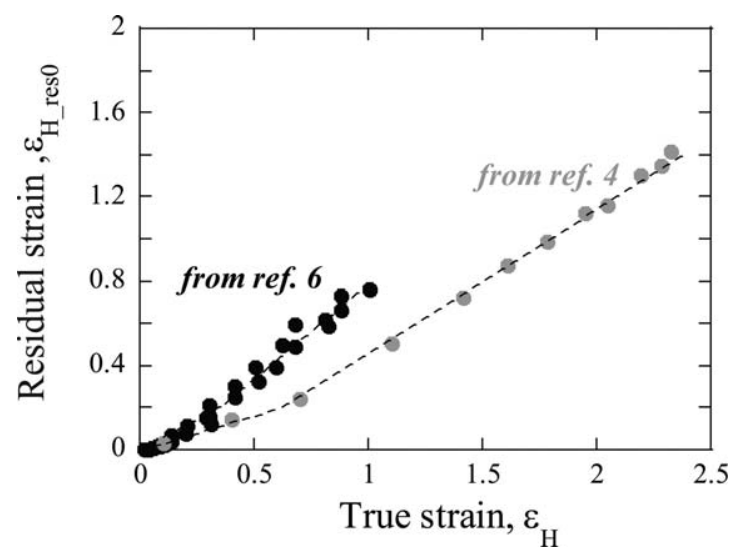

FIGURE 11 Dependence of the residual strain component on the applied true strain for a pure SPP (from ref. 6) and a sPP-EPR copolymer (from ref. 4).

applied overall strain occurs at $\varepsilon_{H}=0.9$ at which the same crystalline form transition takes place. Furthermore, also for s-PP91.5 no change in the residual strain trend can be observed at $\varepsilon_{H}=0.6$ at which the form I to form III transition sets in. The strains at which the recovered strain component relevant to s-PP78 and s-PP91.5 reaches a constant value result to be similar to the strains at which only the crystalline form III is present. ${ }^{5}$

\section{CONCLUSIONS}

Through a test method in which different Zones of a single specimen can be considered independently, the mechanical response of three s-PPs in terms of recovered/residual strain components was investigated up to large strains. Since during the test each Zone undergoes a different strain history, in order to apply the method it has to be verified that the mechanical response of the material is the same irrespective of loading history.

The main finding of this work is that at a critical residual strain value of about 0.2 there is a change in the overall strain dependence of the residual strain irrespective of the syndiotactic pentad [rrrr] content in pure s-PPs. Also in a thermoplastic elastomer in which s-PP hard blocks constitute the physical crosslinks the same critical residual strain was found.

This strain seems to be related to a microstructural change rather to a crystalline form transition.

\section{ACKNOWLEDGMENT}

Financial support by MIUR (PRIN 2008) is gratefully acknowledged.

\section{REFERENCES AND NOTES}

1 B. Lotz, J. C. Wittmann, A. J. Lovinger, Polymer 1996, 37, 4979-4992.

2 C. De Rosa, P. Corradini, Macromolecules 1993, 26, 57115718. 
3 J. Harasawa, H. Uehara, T. Yamanobe, T. Komoto, M. Terano, J. Mol. Struct. 2002, 610, 133-142.

4 F. Deplace, Z. Wang, N. A. Lynd, A. Hotta, J. M. Rose, P. D. Hustad, J. Tian, H. Ohtaki, G. W. Coates, F. Shimizu, K. Hirokane, F. Yamada, Y. Shin, L. Rong, J. Zhu, S. Toki, B. S. Hsiao, G. H. Fredrickson, E. J. Kramer, J. Polym. Sci. Part B: Polym. Phys. 2010, 48, 1428-1437.

5 F. Auriemma, C. De Rosa, J. Am. Chem. Soc. 2003, 125, 13143-13147.

6 Y. Men, G. Strobl, J. Macromol. Sci. B Phys 2001, 40, 775-796.

7 C. D'Aniello, L. Guadagno, C. Naddeo, V. Vittoria, Macromol. Rapid Commun. 2000, 21, 104-108.

8 L. Guadagno, C. D'Aniello, C. Naddeo, V. Vittoria, Macromolecules 2001, 34, 2512-2521.

9 L. Guadagno, C. D'Aniello, C. Naddeo, V. Vittoria, Macromolecules 2002, 35, 3921-3927.
10 B. S. Sprague, J. Macromol. Sci. B Phys. 1973, 8, 157-187.

11 S. L. Cannon, G. B. McKenna, W. O. Statton, J. Polym. Sci. Macromol. Rev. 1976, 11, 209-275.

12 F. Auriemma, O. Ruiz de Ballestreros, C. De Rosa, Macromolecules 2001, 34, 4485-4491.

13 R. Hiss, S. Hobeika, C. Lynn, G. Strobl, Macromolecules 1999, 32, 4390-4403.

14 S. Hobeika, Y. Men, G. Strobl, Macromolecules 2000, 33, 1827-1833.

15 Y. Men, J. Rieger, G. Strobl, Phys. Rev. Lett. 2003, 91, 1-4.

16 Y. Sun, L. Fu, Z. Wu, Y. Men, Macromolecules 2013, 46, 971-976.

17 Y. Wang, Z. Jaing, Z. Wu, Y. Men, Macromolecules 2013, $46,518-522$. 